\title{
Propagation and interaction of nuclei within massive binary system.
}

\section{Jerzy Pabich}

Department of Astrophysics

University of Łódź, Łódź, Poland

E-mail: jpabich@uni.lodz.pl

\section{Włodek Bednarek}

Department of Astrophysics

University of Łódź, Łódź, Poland

E-mail: bednareastro.phys.uni.lodz.pl

\begin{abstract}
We consider propagation of relativistic heavy nuclei in the surrounding of a massive star, a companion in the compact binary system containing also young neutron star (a pulsar) or a second massive star. Relativistic nuclei are injected into the binary system from the pulsar or accelerated at the shock produced in stellar wind collisions. The nuclei follow complicated paths in the stellar magnetic field suffering also multiple photodisintegrations in collisions with thermal photons coming from the massive star. Some secondary neutrons and protons collide with the massive star. We calculate how many nuclei are disintegrated depending on the injection parameters within the binary system. We also show the distribution of secondary neutrons and hadrons on the surface of the massive star. They produce neutrinos which could be observed by the neutrino telescopes.
\end{abstract}

25th Texas Symposium on Relativistic Astrophysics - TEXAS 2010

December 06-10, 2010

Heidelberg, Germany 


\section{The Massive Binary system}

We apply the parameters of the WR star observed within the Cyg X-3 binary system, i.e. the radius $R_{\star}=1.6 \times R_{\odot}$ [1], the effective temperature, $T_{\text {eff }}=1.36 \times 10^{5} \mathrm{~K}$, and typical surface magnetic field, $B_{\star} \sim 10^{2}-10^{3} \mathrm{G}$ [2], the mass loss rate $\dot{M} \sim 0.8-8.0 \times 10^{-5} M_{\odot} \mathrm{yr}^{-1}$, the velocity of the stellar wind at infinity $v_{\infty} \sim(1-5) \times 10^{8} \mathrm{~cm} \mathrm{~s}^{-1}$, and the star rotational velocity $v_{\text {rot }} \sim$ $(0.1-0.2) v_{\infty}$ [3]. For the mentioned above parameters of the massive companion in the Cyg X-3, the value of the Alfven radius in the stellar wind is in the range $1.3 R_{\star}<R_{\mathrm{A}}<2.0 R_{\star}$. It is assumed that the stellar magnetic field has a dipole structure below $R_{\mathrm{A}}$ and a radial structure above $R_{\mathrm{A}}$. The strength of the magnetic field as a function of distance from the center of the massive star can be well approximated by the following equations [2],

$$
B(r) \approx B_{\star} \times \begin{cases}\left(R_{\star} / r\right)^{3}, & R_{\star} \leq r<R_{\mathrm{A}}, \\ R_{\star}^{3} /\left(R_{\mathrm{A}} r^{2}\right), & R_{\mathrm{A}}<r .\end{cases}
$$

\section{Injection of nuclei}

It is assumed that iron nuclei are injected from a point-like source at specific distance $D$ from the surface of the massive star. The distance $D$ can be within a part of the stellar magnetosphere with the dipole or radial magnetic field structure. Such nuclei can be accelerated somewhere in the inner magnetosphere at the pulsar wind zone to energies $\gamma_{\mathrm{Fe}} \approx \chi Z e \Phi_{\mathrm{open}} / m_{\mathrm{Fe}} c^{2} \approx 8 \times 10^{9} \chi B_{12} P_{\mathrm{ms}}^{-2}$, where $m_{\mathrm{Fe}}$ and $Z e$ are the mass and charge of the iron nuclei, $c$ is the velocity of light, and $\Phi_{\text {open }}=\sqrt{L_{\text {rot }} / c}$ is the total electric potential drop across the open magnetosphere, $L_{\text {rot }}$ is the rate of rotational energy lost by the pulsar, $B_{\mathrm{NS}}=10^{12} B_{12} G$ is the surface magnetic field of the pulsar, and $P=10^{-3} P_{\mathrm{ms}}$ is the pulsar period, $\chi$ is not far from unity [4]. Nuclei (e.g. helium, or CNO group) can be also accelerated at the shock which is created as a result of collisions of stellar winds of two massive stars.

\section{Optical depths for nuclei}

Nuclei are injected in a very dense radiation field of the massive star. They can suffer multiple photo-disintegrations of nucleons. In order to check the importance of this process, we calculate the optical depths for photo-disintegration process of iron nuclei with arbitrary Lorentz factors from the injection place at the distance $D$ and propagating along the straight lines at an arbitrary angle $\alpha$,

$$
\tau=\int_{0}^{\infty} \frac{d x}{\lambda\left(\alpha, \gamma_{\mathrm{Fe}}\right)}
$$

The mean free path for dissociation of a single nucleon is calculated from,

$$
\lambda\left(\alpha, \gamma_{\mathrm{Fe}}\right)^{-1}=\int d \Omega \int \sigma_{\text {nucl }}\left(1+\beta_{\mathrm{Fe}} \cos \theta\right) n_{p h} d \varepsilon,
$$

where $\sigma_{\text {nucl }}$ is the cross section for dissociation of a single nucleon taken from [5] (with the modifications [6]), $n_{p h}$ is the differential spectrum of soft photons coming from the massive star within 

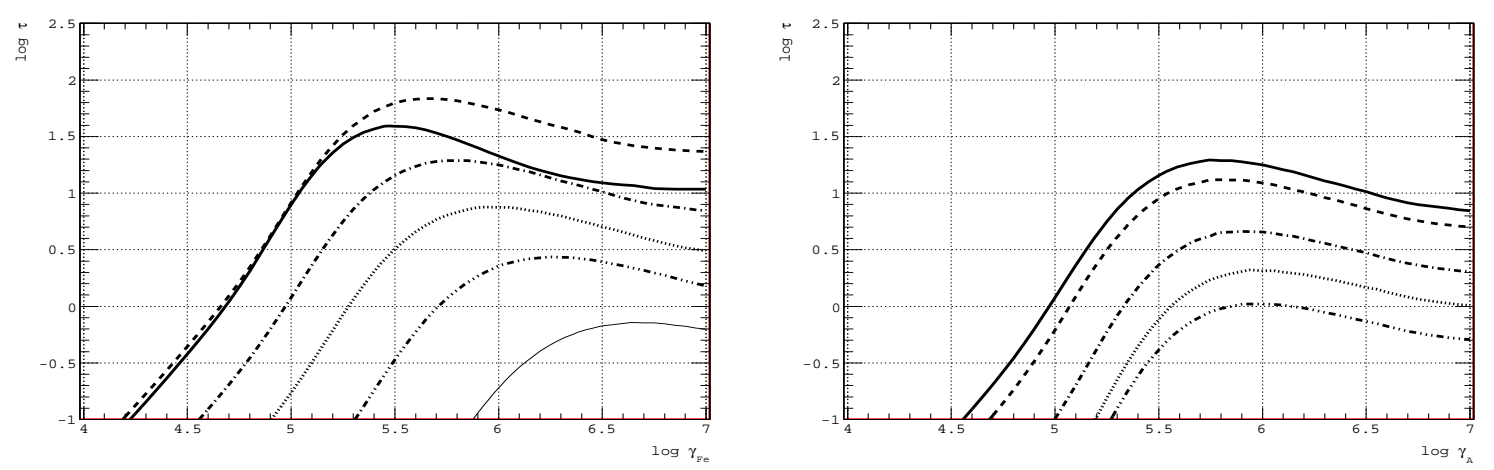

Figure 1: From the left: the optical depth for the Fe nuclei on photo-disintegration process in the radiation field of the massive star (with the radius $R=1.6 R_{\odot}$ and surface temperature $T=1.36 \times 10^{5} \mathrm{~K}$ ) as a function of the Lorentz factor of nuclei and different angles of their injection measured in respect to the direction defined by the injection place and the centre of the massive star: $\alpha=0^{\circ}$ (full curve), $30^{\circ}$ (dashed), $60^{\circ}$ (dot-dashed), $90^{\circ}$ (dotted), $120^{\circ}$ (dot-dot-dot-dashed), and $150^{\circ}$ (thin full). Nuclei propagate radially up to the infinity or to the surface of the star. On the right as above but for nuclei with different mass numbers A = 56 (full curve), 40 (dashed), 16 (dot-dashed), 8 (dotted) and 4 (dot-dot-dot-dashed). The nuclei are injected at the angle $\alpha=60^{\circ}$.

the solid angle $d \Omega$, and $\theta$ is the angle of collision between photon and nuclei. The results are shown in Fig. 1 for different Lorentz factors of Fe nuclei $\left(\gamma_{\mathrm{Fe}}\right)$ and for nuclei with different mass numbers. These optical depths are often above unity for nuclei with Lorentz factors above $\sim 10^{5}$. Therefore, nuclei can suffer severe photo-disintegrations loosing many neutrons and protons.

\section{Propagation of nuclei}

The Larmor radii of nuclei with Lorentz factors $10^{(6-7)}$ can be comparable to the characteristic dimension of the binary system defined by the separation of the stars $D$. Therefore, we have to consider the propagation of hadrons in the vicinity of the massive star taking into account the effects of bending of their paths. The velocity and the coordinates of the charged particle with energy $\mathrm{E}$ as a function of time are given by,

$$
\begin{gathered}
\vec{v}(t+d t)=\frac{q c d t}{E}(\vec{v}(t) \times \vec{B})+\vec{v}(t), \\
\vec{r}(t+d t)=\vec{v}(t+d t) d t+\vec{r}(t) .
\end{gathered}
$$

In our calculations we simulate the propagation and disintegration process of nuclei in the magnetic field and the radiation field of the massive star by using the Monte Carlo method. The structure of the magnetic field in the wind of the massive star is defined in Sect. 1. The example paths of nuclei propagating in the magnetic field of a star for different injection angles and Lorentz factors of nuclei are shown in Fig. 3. We calculate the final mass number of Fe nuclei injected isotropically with different Lorentz factors from a point source in Fig. 2 for two models for the stellar magnetic field. 

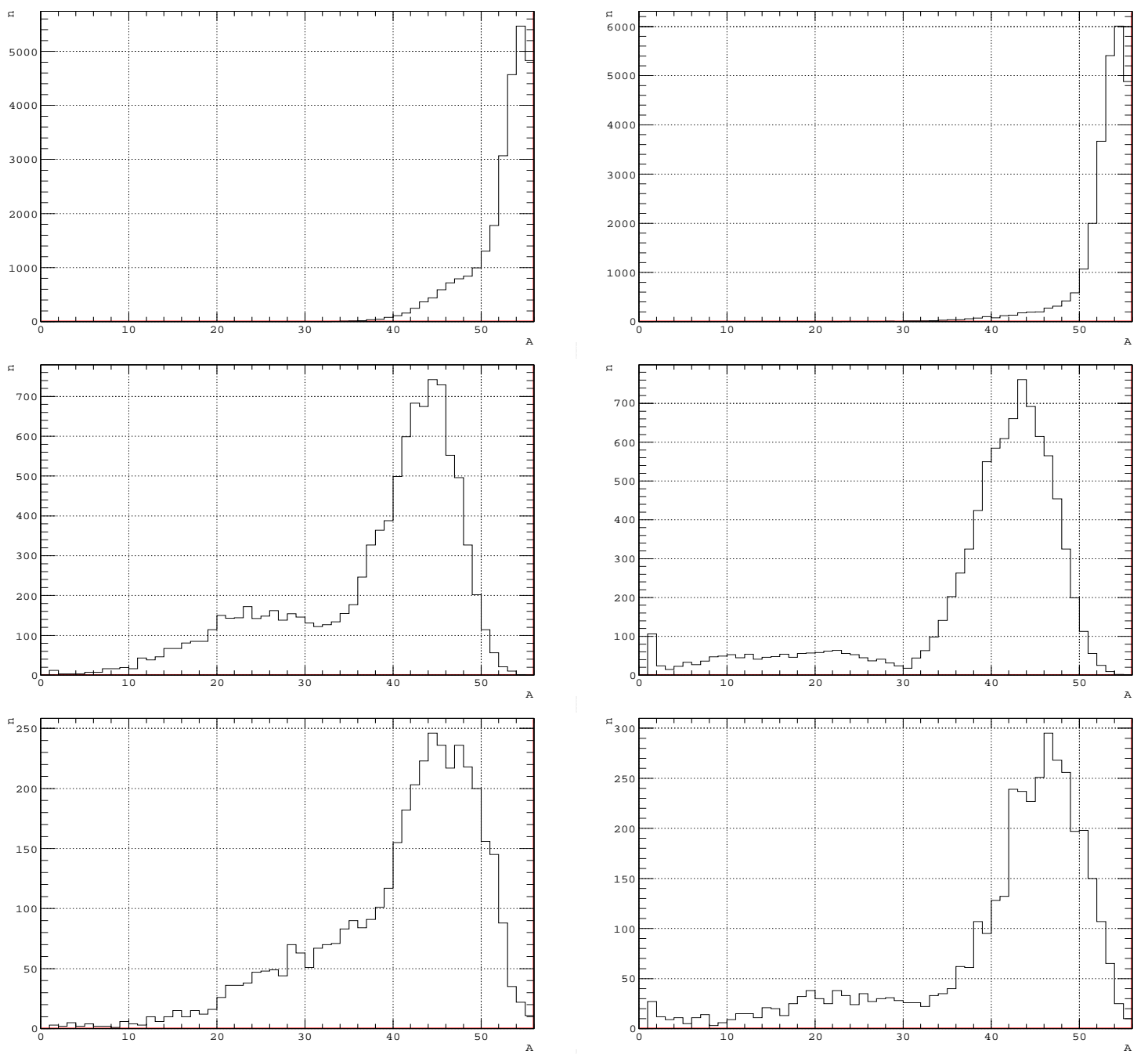

Figure 2: The number of nuclei with the final mass number, A, which survived propagation in radiation field of massive star. Primary Fe nuclei are injected isotropically at the distance $2.0 R_{\star}$ with different Lorentz factors: $\gamma_{\mathrm{Fe}}=10^{5}$ (top), $\gamma_{\mathrm{Fe}}=3 \times 10^{5}$ (middle), $\gamma_{\mathrm{Fe}}=10^{6}$ (bottom). The nuclei propagate in the magnetic field with the structure defined by $R_{\mathrm{A}}=1.3 R_{\star}$ (left) and $R_{\mathrm{A}}=2.0 R_{\star}$ (right).

\section{Hadrons colliding with the massive star}

Significant number of neutrons and protons dissolved from nuclei collide with the stellar surface. Their distributions on the stellar surface are shown in Figs. 4 and 5 for isotropic injection of nuclei with different Lorentz factors and two models for the stellar magnetic field. Note that the distribution patterns strongly depend on the Lorentz factors of primary nuclei. The patterns produced by neutrons and protons are clearly different. These hadrons interact with the matter of the massive star producing neutrinos. Due to the specific collision angles of hadrons with stellar surface, neutrino beams should produce complicated patterns around the binary system. They can be potentially observed only at specific locations in respect to the binary system. 

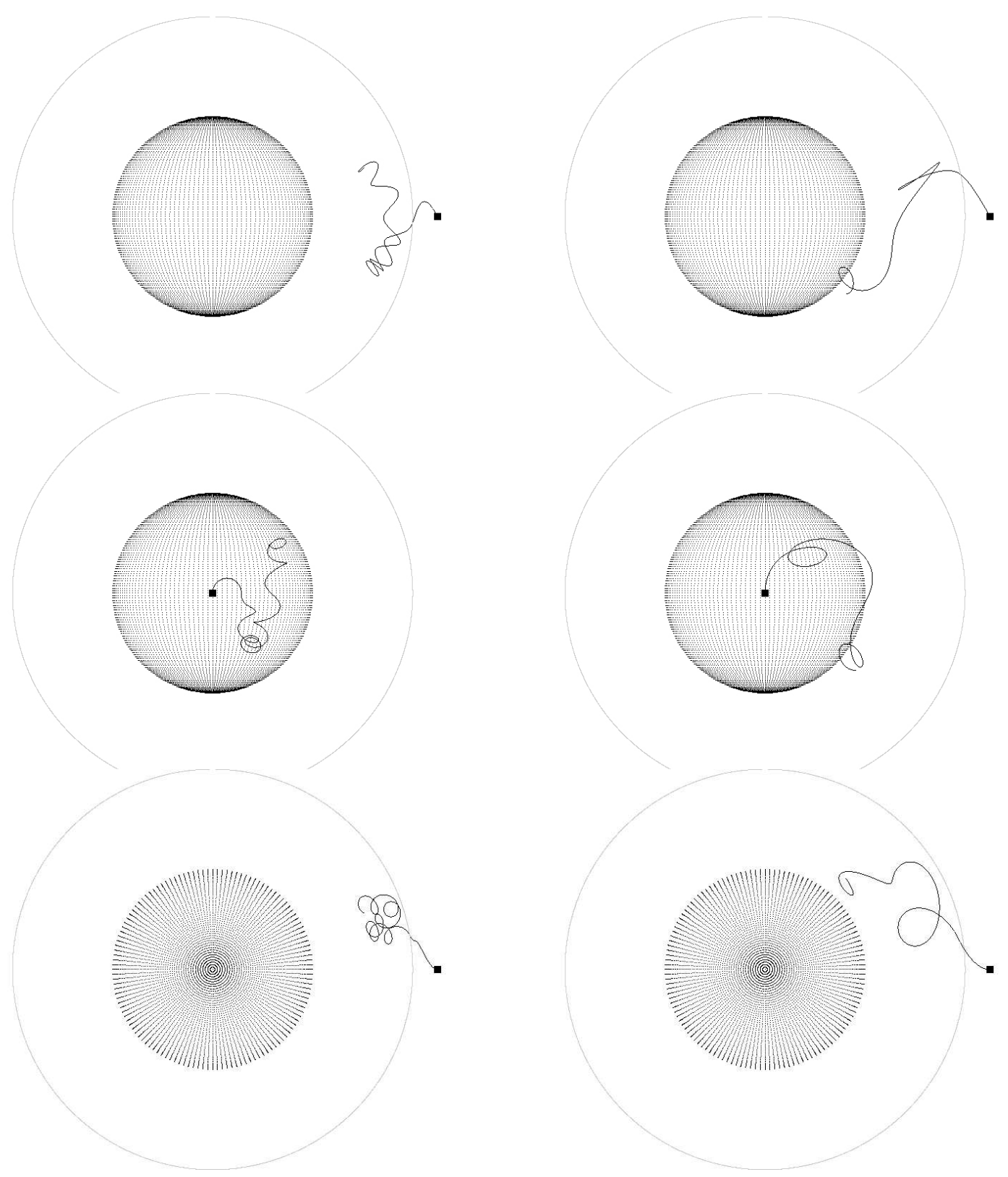

Figure 3: The example paths of primary Fe nuclei with the Lorentz factors $10^{5}$ (on the left) and $3 \times 10^{5}$ (on the right) which are injected at the direction of $\theta=60^{\circ}$ (measured from the plane of the binary system) and $\varphi=0^{\circ}$ (towards the star) from the distance $2.25 R_{\star}$. Nuclei propagate in the magnetic field with the model defined by $R_{\mathrm{A}}=2.0 R_{\star}$ (indicated by the circle): the side view (top), the injection place in front of the star (middle), and from the top (bottom). Protons from disintegration of the primary nucleus also propagate in the magnetic field but neutrons follow the straight lines which can intersect the stellar limb in some cases. 

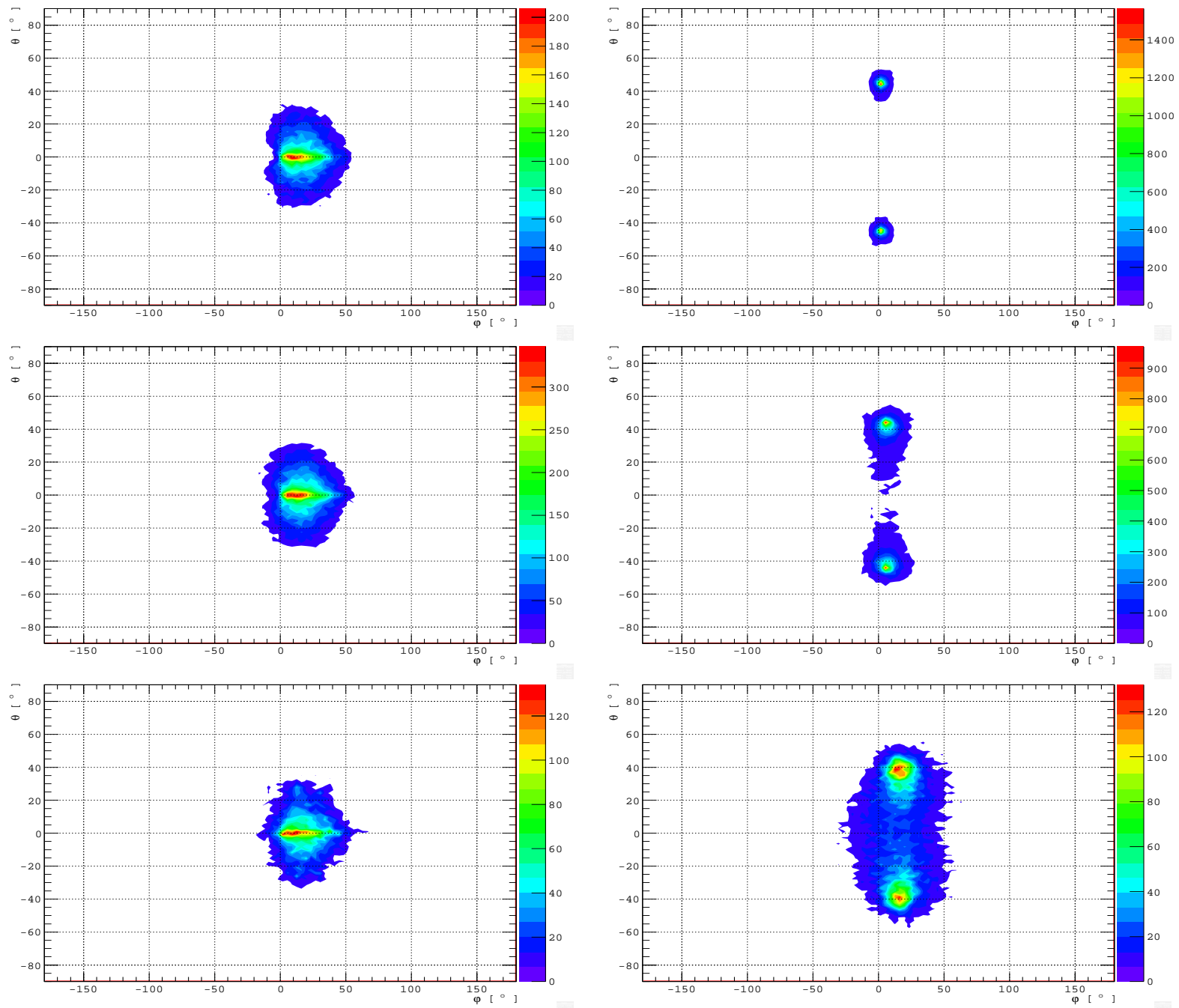

Figure 4: The distribution of collision sites of secondary neutrons, dissolved from primary Fe nuclei propagating in the magnetic field, on the surface of the massive star. Primary Fe nuclei are injected isotropically at the distance $2.0 R_{\star}$ with different Lorentz factors: $10^{5}$ (top), $3 \times 10^{5}$ (middle), $10^{6}$ (bottom). Calculations are made for two models of the magnetic field with Alfven radius: $R_{\mathrm{A}}=1.3 R_{\star}(\mathrm{left})$ and $R_{\mathrm{A}}=2.0 R_{\star}($ right $)$.

\section{Conclusion}

- The efficient disintegration of nuclei has to occur in the case of compact binary systems containing WR type star.

- Nuclei with Lorentz factors for which disintegration occurs follows complicated paths in the magnetic field, which are very characteristic in the vicinity of such stars.

- Significant number of neutrons, hadrons and remnants of partially disintegrated nuclei impinge onto the stellar surface creating characteristic patterns on the stellar surface.

- These hadrons should interact with the matter on the stellar surface producing neutrinos with complicated distribution around the binary system. 

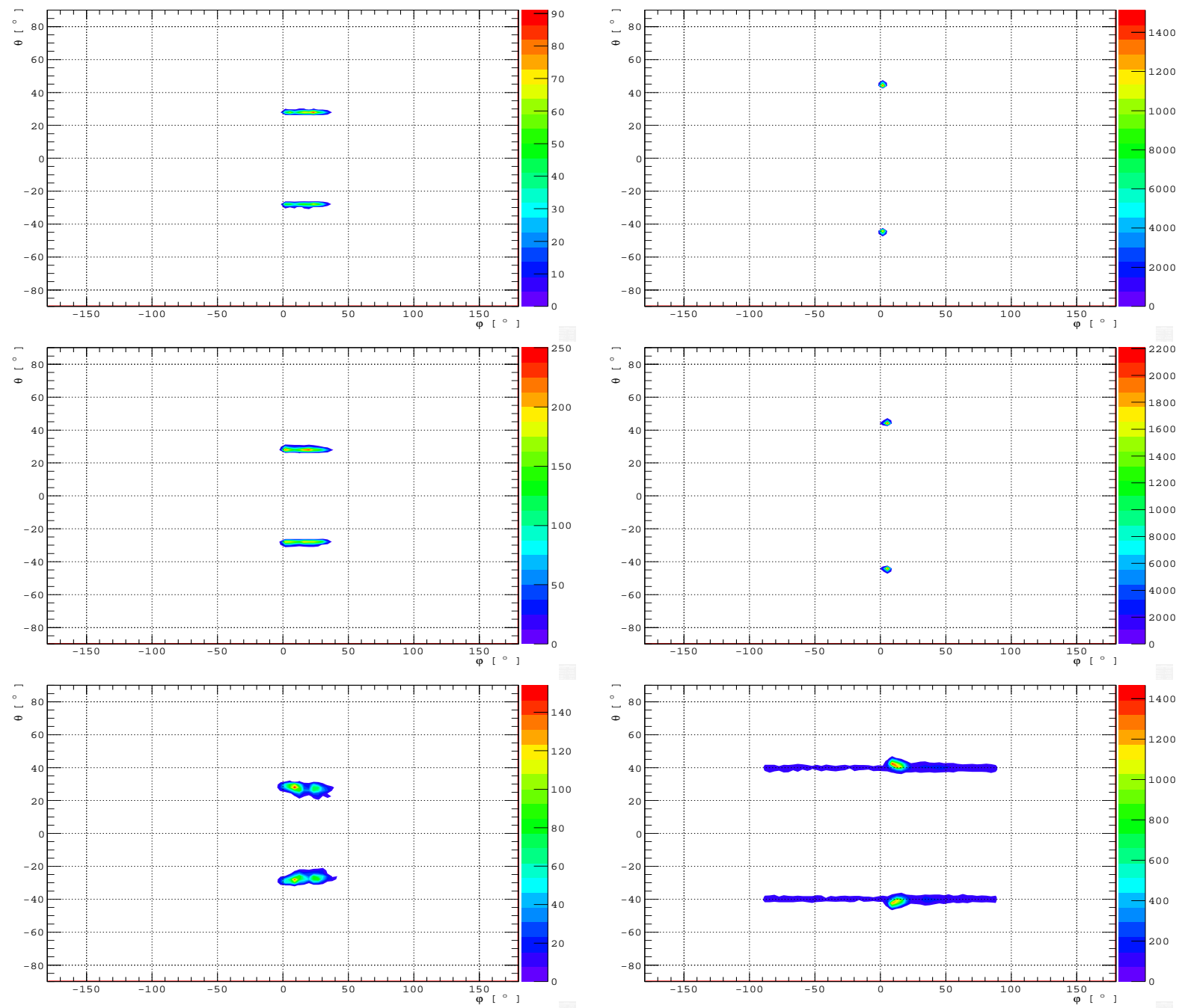

Figure 5: As in Fig. 4 but for secondary protons.

\section{References}

[1] Stark, M., Saia, M., ApJ 587 (2003) L101.

[2] Usov, V.V., Melrose, D.B., ApJ 395 (1992) 575.

[3] van Kerkwijk, M.H., Geballe, T.R., King, D.L. et al., A\&A 314 (2002) 521.

[4] Arons J., Mem.Soc.Ast.Ital. 69 (1998) 989.

[5] Karakuła, S., Tkaczyk, W., APh, 1 (1993) 229.

[6] Protheroe, R.J., Bednarek, W., Luo, Q., APh 9 (1998) 1. 